\title{
Violência obstétrica no período de parto ativo
}

\section{Obstetric violence in the active parturition period \\ Violencia obstétrica en el período de parto activo}

Vitoria Vilas Boas da Silva Bomfim

ORCID: https://orcid.org/0000-0003-4897-0279

Centro Universitário Jorge Amado, Brasil E-mail: pesquisaclinica9@gmail.com Tâmara Araújo Silva

ORCID: https://orcid.org/0000-0003-3898-4669

Centro Universitário Jorge Amado, Brasil

E-mail: enfermeiratamara21@gmail.com

Brenda Tayrine Tavares Souza ORCID: https://orcid.org/0000-0003-4469-3198 Centro Universitário do Distrito Federal, Brasil E-mail: brenndatayrine@gmail.com

Andressa Mayara Nascimento Santos ORCID: https://orcid.org/0000-0002-0942-6054 Faculdade Cesmac do Sertão, Brasil

E-mail: andressa-mayara@outlook.com.br

Maria Clara Teles Cabanelas Macedo ORCID: https://orcid.org/0000-0003-2025-7789 Universidade Salvador, Brasil E-mail: claracabanelas@gmail.com

Maria Dhescyca Ingrid Silva Arruda

ORCID: https://orcid.org/0000-0002-9073-7844

Faculdade São Francisco da Paraíba, Brasil

E-mail: dhescycaingrid20@gmail.com

Angelica Ribeiro do Nascimento Oliveira

ORCID: https://orcid.org/0000-0002-7240-2004

Centro Universitário Maurício de Nassau, Brasil

E-mail: angelicalribeiro.ar19@gmail.com

Lillyan Ranieli Barbosa da Silva

ORCID: https://orcid.org/0000-0002-6768-3147

Centro Universitário Maurício de Nassau, Brasil

E-mail: lillyanrani@hotmail.com

Jade de Oliveira Santana

ORCID: https://orcid.org/0000-0002-2758-0477 Escola Bahiana de Medicina e Saúde Pública, Brasil

E-mail: jadesantana19.2@bahiana.edu.br

Mariana Cunha de Albuquerque

ORCID: https://orcid.org/0000-0001-5366-1150 Universidade Federal de Sergipe, Brasil E-mail: mariana.albcunhas@gmail.com

Marcela Rosa da Silva

ORCID: https://orcid.org/0000-0002-1333-1576 Universidade Luterana do Brasil, Brasil E-mail: marcelasilva@hcpa.edu.br

Gleice Matos Guimarães

ORCID: https://orcid.org/0000-0001-7567-7153

Faculdade Pitágoras Teixeira de Freitas, Brasil

E-mail: gleiceguimaraes64@gmail.com

Lucília da Costa Silva

ORCID: https://orcid.org/0000-0001-9386-5684 Centro Universitário Santo Agostinho, Brasil E-mail: luciliafisio@outlook.com

Natádina Alves Souza Campos

ORCID: https://orcid.org/0000-0001-9970-2405

Universidade Federal do Recôncavo da Bahia E-mail: natadinasouza@gmail.com 


\begin{abstract}
Resumo
Objetivo: O presente estudo promove uma reflexão, baseada em evidências, acerca das formas de violência obstétrica disponíveis na literatura brasileira. Métodos: Trata-se de uma revisão integrativa da literatura realizada através das bases de dados Scientific Electronic Library Online (SciELO), Literatura Latino-Americana e do Caribe em Ciências da Saúde (LILACS), Banco de Dados em Enfermagem (BDENF) e Medical Literature Analysis and Retrieval System Online (MEDLINE). A partir da busca inicial com os descritores e operador booleano definidos, foram encontrados 52 estudos nas bases selecionadas e após aplicar os critérios de inclusão e exclusão, foram selecionados 12 estudos para compor a revisão. Resultados e Discussão: Os locais abordados nos estudos, foram os hospitais públicos e mistos (privados) conveniados ao SUS. A violência obstétrica, termo reconhecido pela Organização Mundial de Saúde (OMS), se firma a partir da apropriação da autonomia da mulher e dos processos reprodutivos, por profissionais de saúde, na forma de um tratamento desumanizado, medicação abusiva ou interferência abusiva dos processos naturais. Ficaram evidenciados, os diversos tipos de violência física, verbal e psicológica, assim como a realização de procedimentos desnecessários, com destaque para o alto índice de cesarianas ocorrendo fora de contexto e expondo a mulher a três vezes mais o risco de morte por parto. Conclusão: E pôde-se observar o grande diferencial da divulgação do que se trata a violência obstétrica e como é imperativo o reforço dos direitos das mulheres, que asseguram um atendimento humanizado, bem como estudos sobre sua abordagem no pré-natal.
\end{abstract}

Palavras-chave: Violência; Parto humanizado; Saúde da mulher; Parto.

\begin{abstract}
Objective: This study promotes an evidence-based reflection on the forms of obstetric violence available in the Brazilian literature. Methods: This is an integrative literature review carried out using the Scientific Electronic Library Online (SciELO), Latin American and Caribbean Literature in Health Sciences (LILACS), Nursing Database (BDENF) and Medical databases. Literature Analysis and Retrieval System Online (MEDLINE). From the initial search with defined descriptors and Boolean operator, 52 studies were found in the selected databases and after applying the inclusion and exclusion criteria, 12 studies were selected to compose the review. Results and Discussion: The places covered in the studies were public and mixed (private) hospitals affiliated to the SUS. Obstetric violence, a term recognized by the World Health Organization (WHO), is based on the appropriation of women's autonomy and reproductive processes by health professionals, in the form of dehumanized treatment, abusive medication or abusive interference with natural processes. The various types of physical, verbal and psychological violence were highlighted, as well as the performance of unnecessary procedures, highlighting the high rate of cesarean sections occurring out of context and exposing the woman to three times the risk of death by childbirth. Conclusion: And it was possible to observe the great difference in the dissemination of what obstetric violence is and how it is imperative to reinforce the rights of women, which ensure humanized care, as well as studies on its approach to prenatal care.
\end{abstract}

Keywords: Violence; Humanizing delivery; Women's health; Parturition.

\title{
Resumen
}

Objetivo: Este estudio promueve una reflexión basada en la evidencia sobre las formas de violencia obstétrica disponibles en la literatura brasileña. Métodos: Revisión integrativa de la literatura realizada utilizando la Biblioteca Científica Electrónica en Línea (SciELO), Literatura Latinoamericana y del Caribe en Ciencias de la Salud (LILACS), Base de Datos de Enfermería (BDENF) y Bases de Datos Médicas. Sistema de Recuperación y Análisis de Literatura en Línea (MEDLINE). De la búsqueda inicial con descriptores definidos y operador booleano, se encontraron 52 estudios en las bases de datos seleccionadas y luego de aplicar los criterios de inclusión y exclusión, se seleccionaron 12 estudios para componer la revisión. Resultados y Discusión: Los lugares cubiertos en los estudios fueron hospitales públicos y mixtos (privados) afiliados al SUS. La violencia obstétrica, término reconocido por la Organización Mundial de la Salud (OMS), se basa en la apropiación de la autonomía de la mujer y los procesos reproductivos por parte de los profesionales de la salud, en forma de tratamiento deshumanizado, medicación abusiva o injerencia abusiva en los procesos naturales. Se evidenciaron los diversos tipos de violencia física, verbal y psicológica, así como la realización de procedimientos innecesarios, destacando la alta tasa de cesáreas ocurridas fuera de contexto y exponiendo a la mujer a tres veces el riesgo de muerte por parto. Conclusión: Y se pudo observar la gran diferencia en la difusión de qué es la violencia obstétrica y cómo es imperativo reforzar los derechos de las mujeres, que aseguran la atención humanizada, así como estudios sobre su abordaje en la atención prenatal.

Palabras clave: Violencia; Parto humanizado; Salud de la mujer; Parto.

\section{Introdução}

A violência contra mulher está muito além do ações que deixam marcas físicas aparentes em seus corpos. Dentre os tipos de violências, relacionadas ao gênero feminino derivada de uma sociedade basicamente patriarcalista e machista, podemos classificá-las em cinco tipos segundo a Lei Maria da Penha $\mathrm{n}^{\circ}$ 11.340/2006: física, psicológica, moral, sexual e patrimonial. Violência contra mulher é considerada pela Organização Mundial de Saúde (OMS), como um agravo sério de 
saúde pública a nível mundial, e que vão além da etnia, nível de escolaridade e socioeconômica, são ações que violam os direitos humanos de uma mulher (Dias, 2015).

A mulher sofre violência muitas vezes em diversos âmbitos e ciclos da vida, seja ela em seu ambiente doméstico, profissional e até mesmo institucional, tendo como exemplo, uma mulher em situação de trabalho de parto ativo em instituição prestadora de serviços de saúde. Segundo os autores de Almeida Silva et al. (2021), um há cada quatro gestantes sofre violência obstétrica, ou seja, $25 \%$. Essa violência está associada em todas as situações que acorrem principalmente durante o trabalho de parto, mas pode ocorrer durante toda a gravidez, no puerpério e até em casos de abortos (Almeida et al., 2018).

De acordo com os autores Zanardo et al. (2017), a violência obstétrica é praticada na rede pública e na rede privada, e está ganhando cada vez mais a mídia com relatos de parturientes que já passaram por essa situação. A violência obstétrica atinge mulheres de todas as classes sociais, brancas, pardas, negras ou indigenas. Mesmo aquelas mães que dizem estar informadas e preparadas para encarar essas circunstâncias. É um momento de muita fragilidade, dor e medo, pelo momento de total desconhecimento de si mesma (de Marco Alves et al., 2018).

O parto humanizado vem ganhando uma grande popularidade ao redor do mundo por conta do seu tratamento individualizado e empático, após uma época em que se privilegiou a técnica operatória despersonalizada da cesária. Nesse sentido, o trabalho de parto humanizado tem como principal finalidade diminuir as relações autoritárias e desiguais entre o profissional de saúde e a parturiente, desenvolvendo a autonomia da paciente através das decisões compartilhadas. Sendo essencial adotar este modelo sobretudo para diminuir a submissão da mulher a procedimentos de resultados duvidosos, que levam a violência obstétrica na maioria das vezes (Santos \& Okazaki, 2012).

Frente ao processo de mudança do paradigma do cuidado ao parto e pós-parto, no quadro de enfermagem há um desempenho crucial, já que são os profissionais que têm um contato direto e contínuo das parturientes. Sendo assim indispensável então, que o conjunto da enfermagem auxilie por instrumentos pertinentes e educação estável, um modo de atentar adequado, caracterizando-o como um exercício autônomo e consciencioso do seu desempenho (Ismael et al., 2020). Diante do exposto o estudo objetiva demonstrar mediante a literatura a violência obstetrica no período de parto ativo.

\section{Metodologia}

Trata-se de uma revisão integrativa da literatura, definida por Souza et al. (2010) como um instrumento que utiliza a síntese de conhecimentos e aplicação de resultados de estudos e/ou pesquisas de um determinado assunto. Realizada através das bases de dados Scientific Electronic Library Online (SciELO), Literatura Latino-Americana e do Caribe em Ciências da Saúde (LILACS), Banco de Dados em Enfermagem (BDENF) e Medical Literature Analysis and Retrieval System Online (MEDLINE), através dos seguintes Descritores em Ciências da Saúde (DeSC): "Violência", "Parto Obstétrico" e "Parto Humanizado". Combinados entre si pelo operador booleano AND. Como critérios de inclusão: artigos disponíveis na íntegra, nos idiomas português, espanhol e inglês, que abordassem a temática, nos últimos cinco anos. Como critérios de exclusão: artigos que não contemplavam o tema e estudos repetidos nas bases de dados.

\section{Resultados e Discussão}

A partir da busca inicial com os descritores e operador booleano definidos, foram encontrados 52 estudos nas bases selecionadas e após aplicar os critérios de inclusão e exclusão, foram selecionados 12 estudos para compor a revisão conforme demonstra o Quadro 1. 
Quadro 1 - Síntese dos principais achados sobre violência obstétrica no período do parto ativo.

\begin{tabular}{|c|c|c|}
\hline $\mathbf{N}$ & Autores (Ano) & Principais achados \\
\hline 1 & Gasperin et al. (2019) & $\begin{array}{l}\text { Trata-se de uma pesquisa exploratória e bibliográfica que buscou retratar convergências } \\
\text { e divergências presentes na violência obstétrica sofridas por mulheres durante o parto. } \\
\text { Por meio desse estudo, foi possível analisar que por ser um assunto amplo e complexo, a } \\
\text { discussão sobre essa temática é complexa e ainda recente, podendo concluir que o } \\
\text { próprio conceito sobre a violência obstétrica ainda não está concretizado por não ter } \\
\text { pesquisas suficientes que possam aprofundar o assunto. }\end{array}$ \\
\hline 2 & dos Santos Niculau et al. (2021) & $\begin{array}{l}\text { Revisão integrativa de Literatura. A violência obstétrica é um aspecto histórico da } \\
\text { assistência ao parto e perfaz um problema de saúde pública. São apontadas como } \\
\text { aspectos culturalmente perpetuados a assistência médica hospitalocêntrica, questões } \\
\text { intrínsecas ao gênero feminino, profissionais que se sentem ordenadores das devidas } \\
\text { necessidades da parturiente, o processo de formação acadêmica, déficit de conhecimento } \\
\text { das pacientes, falta de comunicação e interação entre os profissionais e pacientes, } \\
\text { condições estruturais das instituições de saúde e carga horária excessiva. }\end{array}$ \\
\hline 3 & Oliveira \& Penna (2017) & $\begin{array}{l}\text { Estudo interpretativo com abordagem qualitativa, por meio da análise de discurso. Foi } \\
\text { coletado dados em sete maternidades da rede pública da região Centro-Oeste de Minas } \\
\text { Gerais. Houve uma análise dos discursos de profissionais de saúde e mulheres sobre o } \\
\text { auxílio durante o parto. Conclui-se que a violência obstétrica ainda é presente na sala de } \\
\text { parto, impedindo recuperar a autonomia das suas escolhas quanto a via de parto e } \\
\text { dificultando no tratamento humanizado. }\end{array}$ \\
\hline 4 & Ribeiro et al. (2021) & $\begin{array}{l}\text { Trata-se de um estudo de revisão integrativa da literatura em um corte temporal de } 12 \\
\text { anos. Foram utilizadas as bases de dados LILACS, Scielo e PUBMED para a busca e } \\
\text { seleção de artigos. A violência obstétrica está relacionada à realização de procedimentos } \\
\text { sem o consentimento da gestante em qualquer situação em que não há previsão legal para } \\
\text { sustentar a execução da ação de saúde. }\end{array}$ \\
\hline 5 & Estumano et al. (2017) & $\begin{array}{l}\text { Revisão de Literatura de caráter qualitativo no objetivo de recolher informações sobre a } \\
\text { violência obstétrica. Foi coletado dados por meio do levantamento bibliográfico através } \\
\text { da busca eletrônica de artigos indexados em bases de dados como LILACS, SciELO, a } \\
\text { Biblioteca Virtual em Saúde (BVS) e BDENF (Base de dados em Enfermagem). A } \\
\text { discussão sobre a violência obstétrica ainda é pouco presente na sociedade. Pois, a falta } \\
\text { de conhecimento das gestantes facilita o risco e a vulnerabilidade sofrida por essas } \\
\text { mulheres no pré-parto e no momento do parto. Sendo assim, é importante enfatizar que } \\
\text { toda mulher tem direito a um atendimento digno, respeitoso e de qualidade durante o } \\
\text { período de gestação. }\end{array}$ \\
\hline 6 & Rocha \& Ferreira (2020) & $\begin{array}{l}\text { Uma revisão integrativa acerca da escolha da via de parto no modelo de atenção } \\
\text { obstétrica brasileira. A busca resultou em } 538 \text { documentos. Após as análises, foram } \\
\text { selecionados } 15 \text { documentos para compor a revisão. Concluindo que o medo da dor e o } \\
\text { medo de sofrer violência na hora do parto apareceram nos discursos como fatores } \\
\text { negativos do parto normal, o que reflete o impacto da violência obstétrica na saúde e } \\
\text { reforça a importância da informação no processo de gestar e parir. }\end{array}$ \\
\hline 7 & Lansky et al. (2019). & $\begin{array}{l}\text { Estudo transversal multicêntrico e multimétodos com componente quantitativo e } \\
\text { qualitativo. Foram utilizados os dados coletados em entrevistas após o parto, entre junho } \\
\text { de } 2015 \text { a janeiro de } 2017 \text {. O questionário pós-parto foi aplicado por meio telefônico, } \\
\text { contendo perguntas sobre a sua experiência no parto. Visto que a violência obstétrica é } \\
\text { uma situação de importância na saúde da mulher e da criança e influencia a cultura e a } \\
\text { percepção sobre o parto pela sociedade, relatos de } 12,6 \% \text { das mulheres entrevistadas } \\
\text { reflete o desconhecimento e subestimação do problema. Sendo importante a exposição } \\
\text { Sentidos do Nascer buscar incentivar o parto normal para promover a saúde e melhorar a } \\
\text { experiência de parir e nascer no País. }\end{array}$ \\
\hline 8 & de Figueiredo Júnior et al. (2021) & $\begin{array}{l}\text { O estudo foi do tipo qualitativo descritivo com o intuito de realizar um levantamento dos } \\
\text { principais tipos de violência obstétrica que ocorrem durante a assistência ao parto em } \\
\text { âmbito hospitalar através de uma Revisão Integrativa da Literatura. É necessário o } \\
\text { treinamento de profissionais da saúde para promover um atendimento humanizado e } \\
\text { adequado para cuidados de saúde da mulher. }\end{array}$ \\
\hline 9 & Fernandes et al. (2019) & $\begin{array}{l}\text { Tratou-se de um estudo com visão retrospectiva, de abordagem qualitativa, o método foi } \\
\text { composto por Narrativas de Vida, sendo o estudo realizado em uma maternidade do Rio } \\
\text { de Janeiro com } 12 \text { mulheres com diagnóstico de feto anencéfalo. Após análise } \\
\text { compreensiva e comparativa dos dados, a violência obstétrica surgiu predominantemente } \\
\text { na forma de julgamento moral das escolhas das mulheres, má assistência, abusos, } \\
\text { utilização de jargões, entre outras. Concluindo que nessas experiências permeadas por } \\
\text { sofrimentos e perdas, a violência obstétrica amplia a situação de vulnerabilidade das } \\
\text { mulheres, havendo a necessidade de um debate mais aprofundado. }\end{array}$ \\
\hline
\end{tabular}




\begin{tabular}{|l|l|l|}
\hline \multirow{1}{*}{10} & de Souza et al. (2018) & $\begin{array}{l}\text { Método a revisão integrativa da literatura.Para a elaboração desta revisão integrativa, } \\
\text { formulou-se a seguinte questão de pesquisa: Qual o conhecimento científico produzido } \\
\text { na literatura sobre a violência obstétrica e se a mesma é abordada durante o pré-natal? } \\
\text { Evidenciou-se que a violência obstétrica é ainda muito presente na assistência ao parto, e } \\
\text { que um momento que deveria ser de confiança entre a equipe de saúde e a parturiente, } \\
\text { torna-se para a mulher um perído marcado por medos, anseios e sentimentos negativos. } \\
\text { E pode-se observar a importância da divulgação do que é violência obstétrica, dos } \\
\text { direitos das mulheres, que se assegurem seus direitos de um atendimento humanizado e } \\
\text { de qualidade, bem como estudos sobre sua abordagem no pré-natal. }\end{array}$ \\
\hline \multirow{5}{*}{11} & Moura et al. (2018) & $\begin{array}{l}\text { Revisão integrativa da literatura, realizada entre os meses de maio a junho de 2017, nas } \\
\text { bases de dados online Scientific Electronic Library Online (SciELO) e na Literatura } \\
\text { Latino-Americana e do Caribe em Ciências da Saúde (LILACS). Foram detectados } 30 \\
\text { artigos sendo utilizados 09 artigos. Para prevenir a violência obstétrica faz-se necessário } \\
\text { uma assistência de enfermagem e um ambiente que proporcione a autonomia da mulher } \\
\text { gestante. }\end{array}$ \\
\hline Vilela et al. (2021) & $\begin{array}{l}\text { Foi realizada Estimativa Rápida Participativa. O local de estudo foram incluídos os } \\
\text { hospitais públicos e mistos (privados conveniados ao SUS). Com o parto e nascimento } \\
\text { nas maternidades da Rede Cegonha ressalta-se a desafiante tarefa de se fazer movimento, } \\
\text { promovendo articulação local e ampliando a capacidade de reflexão e ação de gestores } \\
\text { do SUS e equipes das maternidades. Nesse sentido pode-se afirmar que, enquanto ação } \\
\text { transformadora em serviços de cuidado obstétrico e neonatal, o projeto de avaliação das } \\
\text { maternidades abriu novas perspectivas e produziu aprendizados. }\end{array}$ \\
\hline
\end{tabular}

Fonte: Autores (2021).

Até o final do século XVIII o parto era um evento feminino, doméstico, natural, assistido por parteiras. No final do século XIX iniciou um movimento de mudança na tentativa de controle do evento biológico pela obstetrícia para tornar o parto uma prática médica. O parto e nascimento que antes eram vistos como um processo natural e feminino começa a ser tratado como uma prática de risco e patológica protagonizado então por médicos do gênero masculino e intervenções, deixando a mulher como coadjuvante no processo de parturição (Zanardo et al., 2017)

No século XX acelerou a hospitalização do parto chegando ao final do século com cerca de $90 \%$ dos partos institucionalizados. Com a chegada das tecnologias e a necessidade do imediatismo da sociedade moderna, o parto fisiológico foi substituído por intervenções para acelerar o processo de nascimento. Com a necessidade de agilidade, rapidez e falta de paciência em assistir um trabalho de parto surge a violência obstétrica (Russo et al., 2019). Percebe-se então que o surgimento desse tema é recente, o que reforça os dados encontrados neste estudo quando relata que o termo "violência obstétrica" ainda não está concretizado (Rocha \& Ferreira, 2020).

Segundo a Organização Mundial de Saúde violência é um grau de imposição que causa dor e/ou sofrimento evitáveis. Sendo então a violência obstétrica um tipo específico de violência contra a mulher. A violência obstétrica é conhecida como a apropriação do corpo da mulher durante a fase reprodutiva, pela equipe de saúde que assiste a mulher durante o trabalho de parto e parto de maneira desumana (Ribeiro et al., 2021).

Se caracteriza pelo excesso de intervenções sem evidências científicas, da patologização do parto, de diálogos agressivos, maus tratos físicos e psicológicos e da falta de informação nos procedimentos realizados o que faz com a mulher perca o poder de decisão e o protagonismo do parto trazendo desfechos negativos para ela e para a criança. Com esse estudo podemos evidenciar os dados encontrados na presente pesquisa ao referir-se que ainda está presente nas salas de parto do Brasil (Serra, 2018; Fernandes et al.,2019).

Nesse sentido as mulheres sofrem diversas violências nos serviços de saúde, porém muitas vezes por desconhecerem seus direitos, pela desinformação e pelo medo da exposição frente aos profissionais de saúde essa violência não é relatada e algumas vezes nem mesmo percebida pelas parturientes e seus familiares (Zanardo et al., 2017).

Segundo os autores Estumano et al (2017) a violência obstétrica pode ser dividida em categorias: Abuso físico são os procedimentos realizados sem evidências científicas, como toques vaginais de repetição, cesáreas e episiotomias 
desnecessárias, mobilização física e práticas dolorosas sem analgesia adequada. Iimposição de intervenções não consentidas: são dadas informações parciais ou distorcidas para convencer a mulher a realizar a intervenção como episiotomias sem autorização, recusa aceitação do plano de parto, indução de cesárea por motivos duvidosos, como circular de cordão, pósdatismo.

O cuidado não privativo em enfermarias de trabalho de parto coletivas sem a mínima privacidade entre os leitos cuidado indigno e abuso sexual são retratados no estudo de Lansky et al. (2019) bem como, a comunicação agressiva, desprezo da dor e dos pedidos de ajuda e humilhação de caráter sexual e discriminação baseada em certos atributos: tratamento diferencial com base em atributos considerados positivos, como estado civil, cor, classe social, depreciando as que têm atributos considerados negativos.

Um estudo que visou compreender o contexto da violência obstétrica no Brasil concluiu que a violência ocorre em salas de parto tanto do setor privado como do setor público, porém com categorias diferentes. No setor privado há uma incidência maior da categoria de imposição de intervenções não concedidas evidenciada pelo número de cesarianas desnecessárias nesses serviços visando o benefício para o hospital. Já nos serviços públicos percebe-se a presença da violência em todas as categorias descritas anteriormente com o objetivo de acelerar o parto (Oliveira \& Penna, 2017).

Faz-se necessário estratégias que possibilitem a autonomia das mulheres durante o trabalho de parto por meio de ações educativas que possibilitem o reconhecimento dessas violências que muitas vezes é considerado algo normal. A mulher necessita ter um conhecimento sobre os tipos de violências para que a mesma saiba intervir a fim de reduzir os casos dessa violação durante o parto e que seus direitos sejam respeitados (Vilela et al., 2021; dos Santos Niculau et al., 2021).

A equipe deve prestar os serviços durante o trabalho de parto de forma humanizada e integral para que seja criado um vínculo entre paciente e profissional que resulte em confiança e que seja um momento saudável pois essas violências contra a parturiente pode gerar traumas, medos, insegurança e posteriormente ocasionar complicações durante o parto (de Souza et al., 2018; Gasperin et al., 2019).

Desta forma é possível perceber que a violência obstétrica ainda é um desafio a ser superado nos serviços de saúde. Seu amplo conceito, a falta de informação dos usuários dos serviços de saúde, o medo de represálias na assistência, são fatores que dificultam a percepção e o conceito desse tema. Pesquisas com alto rigor metodológico poderão ser úteis para a criação de evidências científicas na assistência em saúde prestada às gestantes, promovendo uma melhor definição do termo "violência obstétrica" e na criação de ações para combatê-la.

Deve incluir a interpretação dos autores sobre os resultados obtidos e sobre suas principais implicações, a comparação dos achados com a literatura, as limitações do estudo e eventuais indicações de caminhos para novas pesquisas.

\section{Considerações Finais}

A violência obstétrica é uma situação de importância para a saúde da mulher e da criança e influencia a cultura e a percepção sobre o parto pelas sociedades, a práticas assistenciais obsoletas se concentra na maioria das vezes na população de menor renda. Afeta as mulheres de maneira diferenciada na sociedade brasileira, para um parto verdadeiramente ativo é necessário uma mulher ativa, um acompanhante (o pai do bebê ou outro por ela escolhido), um bebê e alguém que fique ao lado apenas verificando se tudo está bem, sem intervir no processo natural do nascimento,o corpo da mulher já vem preparado para o parto,todo mulher consegue parir,é permitindo que a mulher adote todas as posições que lhe parecerem confortáveis, se possibilitamos a liberdade de movimento e ações, se o ambiente do parto for propício para essa liberdade, mãe e bebê encontrarão a fórmula para a travessia que eles têm que fazer. Ainda assim faz-se necessários novos estudos acerca da temática a fim de pautar as práticas assistênciais e melhorar o cuidado prestado a gestante vítima de violência 


\section{Referências}

Almeida, M. M., da Costa Cardoso, F. J., da Costa, A. C. M., de Macêdo, W. B. S., Pessôa, R. M. C., Azevêdo, C. A. S., ... \& Penha, A. S. (2018). Vivência e saberes das parturientes acerca da violência obstétrica institucional no parto. Revista Eletrônica Acervo Saúde/Electronic Journal Collection Health ISSN, 2178, 2091.

de Almeida Silva, S., de Carvalho Garcia, L. P., Alves, T. H. E., \& Inácio, M. C. (2021). Violência obstétrica. Revista de Educação Popular, $20(1)$, 4-13.

de Figueiredo Júnior, A. M., Moraes, A. D. S. C., da Silva, C. L. T., de Souza Cobel, J., Pinho, K. P., Pimentel, M. D. J. E., \& da Rocha, S. M. G. (2021). As faces da violência obstétrica no âmbito hospitalar. Revista Eletrônica Acervo Científico, 23, e7043-e7043.

de Marco Alves, L., Siqueira, F. P. C., \& Cardoso, F. M. Violência obstétrica: investigação da prevalência de práticas parturitivas. Revista Eletrônica Acervo Saúde/Electronic Journal Collection Health ISSN, 2178, 2091.

de Souza, A. Q., Marchiori, M. R. C. T., Ambrós, E. L., Rambo, D. C., Pizolotto, A. L. Z., Segabinazi, A. D., \& Feldns, A. C. (2018). A Violência obstétrica e a abordagem no pré-natal: uma revisão integrativa. Revista Eletrônica Acervo Saúde/Electronic Journal Collection Health ISSN, $2178,2091$.

Dias, M. B. (2015). Lei Maria da penha. São Paulo: Ed. Revistas dos Tribunais.

dos Santos Niculau, D., Feitoza, C. S. V., \& Menezes, M. O. (2021). Reflexões Sobre A Violência Obstétrica No Brasil: Aspectos Culturais. Caderno de Graduação-Ciências Biológicas e da Saúde-UNIT-SERGIPE, 6(3), 95-95.

Estumano, V. K. C., de Melo, L. G. D. S., Rodrigues, P. B., \& do Rêgo Coelho, A. C. (2017). Violência obstétrica no Brasil: casos cada vez mais frequentes. Revista Recien-Revista Científica de Enfermagem, 7(19), 83-91.

Fernandes, I. B., Bento, P. A. D. S. S., \& Xavier, R. B. (2019). Experiências de mulheres no gestar e parir fetos anencéfalos: as múltiplas faces da violência obstétrica. Interface-Comunicação, Saúde, Educação, 23, e170757.

Gasperin, H. G., da Silva, S. E., Ribas, M. C., \& Leandro, J. A. (2019). Violência obstétrica: questões conceituais a partir de um estudo bibliográfico na plataforma SciELO Brasil. Revista Stricto Sensu, 4(2).

Ismael, F. M., Souza, G. K. R., Esteves, N. S., \& de Andrade Aoyama, E. (2020). Assistência De Enfermagem Na Prevenção Da Violência Obstétrica. Revista Brasileira Interdisciplinar de Saúde.

Lansky, S., Souza, K. V. D., Peixoto, E. R. D. M., Oliveira, B. J., Diniz, C. S. G., Vieira, N. F., ... \& Friche, A. A. D. L. (2019). Violência obstétrica: influência da Exposição Sentidos do Nascer na vivência das gestantes. Ciência \& Saúde Coletiva, 24, 2811-2824.

Moura, R. C. D. M., Pereira, T. F., Rebouças, F. J., Costa, C. D. M., Lernades, A. M. G., Silva, L. K. A. D., \& Rocha, K. D. M. M. D. (2018). Cuidados de enfermagem na prevenção da violência obstétrica. Enferm. foco (Brasília), 60-65.

Oliveira, V. J., \& Penna, C. M. D. M. (2017). O discurso da violência obstétrica na voz das mulheres e dos profissionais de saúde. Texto \& ContextoEnfermagem, 26(2).

Ribeiro, K. G., Ribeiro, T. C. D. S. M., Dias, R. D. S. F., Neta, M. E., Leite, M. G., Silva, K. S., ... \& da Silva Junior, R. F. (2021). Caracterização da violência obstétrica na produção científica: uma revisão integrativa. Revista Eletrônica Acervo Saúde, 13(4), e6604-e6604.

Rocha, N. F. F. D., \& Ferreira, J. (2020). A escolha da via de parto e a autonomia das mulheres no Brasil: uma revisão integrativa. Saúde em Debate, 44, 556568.

Russo, J., Nucci, M., Silva, F. L., \& Chazan, L. K. (2019). Escalando vulcões: a releitura da dor no parto humanizado. Mana, 25(2), 519-550.

Santos, I. S., \& Okazaki, E. L. F. J. (2012). Assistência de enfermagem ao parto humanizado. Rev Enferm UNISA [periódico na Internet], 13(1), 64-8.

Serra, M. C. D. M. (2018). Violência obstétrica em (des) foco: uma avaliação da atuação do Judiciário sob a ótica do TJMA, STF e STJ.

Souza, M. T. D., Silva, M. D. D., \& Carvalho, R. D. (2010). Revisão integrativa: o que é e como fazer. Einstein (São Paulo), 8(1), $102-106$.

Vilela, M. E. D. A., Leal, M. D. C., Thomaz, E. B. A. F., Gomes, M. A. D. S. M., Bittencourt, S. D. D. A., Gama, S. G. N. D., \& Lamy, Z. C. (2021). Avaliação da atenção ao parto e nascimento nas maternidades da Rede Cegonha: os caminhos metodológicos. Ciência \& Saúde Coletiva, 26, 789-800.

Zanardo, G. L. D. P., Uribe, M. C., Nadal, A. H. R. D., \& Habigzang, L. F. (2017). Violencia obstétrica en Brasil: una revisión narrativa. Psicologia \& sociedade, 29. 